\title{
FUZZY SPATIAL RANKS FOR OBJECT RECOGNITION ACROSS ILLUMINATION CHANGES
}

\author{
Damien Muselet, Ludovic Macaire \\ Laboratoire LAGIS UMR CNRS 8146 \\ Universite des Sciences et Technologies de Lille \\ Cite Scientifique - Batiment P2 - 59655 Villeneuve d'Ascq - FRANCE \\ dm@i3d.univ-lille1.fr - ludovic.macaire@univ-lille1.fr
}

\begin{abstract}
In this paper, we propose an original scheme to retrieve among all the target images of a database, those which contain the same object as that represented by the query image, these images being acquired under different illumination conditions. Rather than considering the color vectors of the pixels to characterize the images, we propose to introduce and exploit the concept of spatial ranks of CCD sensor responses. Indeed, these values are preserved in case of illumination changes and they take into account both the colors of the pixels and the spatial interactions between them in the image. Since we can not determine these ranks from a color image, we propose to estimate their probabilities of occurrences thanks to fuzzy functions. These probabilities are used by our object recognition scheme whose effectiveness is assessed with a public database that contains images of objects acquired under different illuminations.
\end{abstract}

\section{INTRODUCTION}

\subsection{Object recognition across illumination changes}

In this paper, we propose an original scheme to retrieve among all the target images of a database, those which contain the same object as that represented by the query image, these images being acquired under different illumination conditions (see figure 1).

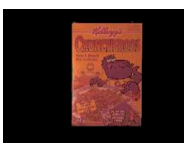

(a)

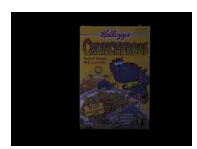

(b)

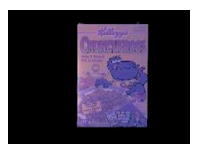

(c)

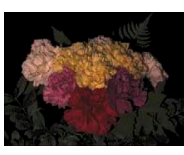

(d)
Fig. 1. As the images (a), (b) and (c) contain the same object observed under different illuminations, they are similar. As the image (d) contains another object, the pairs of images $((\mathrm{a}),(\mathrm{d})),((\mathrm{b}),(\mathrm{d}))$ and $((\mathrm{c}),(\mathrm{d}))$ constitute pairs of different images.
Since the color vector of a pixel $P$, denoted $\mathbf{c}(P)=$ $\left[c^{R}(P), c^{G}(P), c^{B}(P)\right]^{T}$, is not only a measure of the reflectance properties of the elementary surface of the object projected onto the pixel $P$ but also a function of both the camera and the illumination, the color histogram of an image is very sensitive to these parameters. Therefore, many authors propose to characterize the images by histograms which are invariant to illumination changes $[1,2]$. The determination of these invariant color histograms is based on illumination change models which describe the variations of colors caused by any illumination changes. Most of these models try to estimate these variations thanks to linear transformations and are consequently constraint to use very restrictive assumptions about the camera and the illumination. That's the reason why object recognition based on the intersection between these invariant color histograms generally performs poorly $[1,2]$.

Rather than considering the color vectors to define complex illumination change models, we propose to exploit the ranks of sensor responses which respect interesting properties in case of illumination changes.

\subsection{Ranks of sensor responses}

Within a color image $\mathbf{I}$, we associate with each pixel $P$, a vector denoted $\mathbf{x}(P)=\left[x^{R}(P), x^{G}(P), x^{B}(P)\right]^{T}$ whose coordinates $x^{k}(P), k \in\{R, G, B\}$, are the responses of the acquisition CCD camera sensors to the color stimulus reflected by an elementary surface and projected onto the pixel $P$.

For each CCD sensor, the pixels are sorted in the increasing order of these responses and are associated with a rank measure, so that the rank measure is set to 0 for the first ordered pixels, and set to 1 for the last ordered pixels. The rank measure $\mathcal{R}^{k}[\mathbf{I}](s)$ of the CCD sensor response $s$ is the rank of the pixels associated with this response for the $k^{t h} \mathrm{CCD}$ sensor and is expressed as :

$$
\mathcal{R}^{k}[\mathbf{I}](s)=\frac{\operatorname{Card}\left\{Q \in \mathbf{I} / x^{k}(Q) \leq s\right\}}{\operatorname{Card}\{Q \in \mathbf{I}\}}, k \in\{R, G, B\},
$$


where $Q$ are the pixels which represent the object in the image I.

Finlayson shows that the ranks $\mathcal{R}^{k}[\mathbf{I}]\left(x^{k}(P)\right)$ of the sensor responses are invariant to illumination changes [3]. Therefore, for each pixel, he proposes to estimate the ranks of the three CCD sensor responses from the three color component levels of this pixel and to characterize it by these estimated ranks. Nevertheless, we have shown experiments which reveal that the intersection between histograms of so estimated ranks does not provide very satisfying results of object recognition in case of illumination changes [4].

Two main reasons explain these results. First, the ranks of the CCD sensor responses associated with a pixel $P$ have been assumed to be equal to the ranks of the color component levels of this pixel $P$ in the image. We have shown that this assumption is not verified in practice [4]. Secondly, the ranks of the sensor responses do not take into account any spatial information. Nevertheless, in order to improve the quality of object recognition results, it is essential to analyze both the colors of the pixels and the spatial interactions between them in the image.

\subsection{Paper overview}

The main originality of our approach is to cope with the problem of object recognition under illumination changes by combining both color and spatial information. For this purpose, we introduce, in the second section, the concept of spatial ranks of sensor responses. We show that these spatial ranks are preserved in case of illumination changes.

Since we cannot exactly determine these spatial ranks, we propose to estimate their probabilities of occurrence by introducing the fuzzy spatial rank concept in the third section. Then, we determine the histogram of fuzzy spatial ranks to characterize the image for object recognition.

In the fourth section, the effectiveness of our object recognition scheme is assessed with a public database that contains images of objects acquired under different illuminations.

\section{SPATIAL RANKS OF SENSOR RESPONSES}

\subsection{Co-occurrence matrices}

A color image I can be separated into three color component images $I^{k}, k \in\{R, G, B\}$, where each pixel $P$ is characterized by one color component level $c^{k}(P)$.

Let us denote $M^{k, k}[\mathbf{I}]$ the co-occurrence matrix which characterizes the local spatial interaction between the levels of the pixels within the color component image $I^{k}$. This co-occurrence matrix can be considered as an array of cells indexed by color component levels. The cell $M^{k, k}[\mathbf{I}]\left(u, u^{\prime}\right)$ indicates the number of times that, in the image $\mathbf{I}$, a pixel $P^{\prime}$ whose level $c^{k}\left(P^{\prime}\right)$ is equal to $u^{\prime}$, is located in the 8neighborhood of a pixel $P$ whose level $c^{k}(P)$ is equal to $u$. This number is normalized by the total number of cooccurrences so that the matrix does not depend on the number of pixels which represent the object.

The relationships between levels of neighboring pixels within the three color component images are represented by the three matrices $M^{R, R}[\mathbf{I}], M^{G, G}[\mathbf{I}]$ and $M^{B, B}[\mathbf{I}]$.

\subsection{Spatial ranks of color component levels}

We define the spatial rank $\mathrm{SR}^{k}[\mathbf{I}](l)$ of the level $l$ within the color component image $I^{k}$ as the sum of the cells of the cooccurrence matrix $M^{k, k}[\mathbf{I}]$ that represent the spatial interactions between pixels characterized by levels ranging from 0 to $l$ :

$$
\mathrm{SR}^{k}[\mathbf{I}](l)=\sum_{u=0}^{l} \sum_{v=0}^{l} M^{k, k}[\mathbf{I}](u, v), k=R, G, B .
$$

This spatial rank increases with respect to $l$ and ranges from 0 to 1 since the matrices $M^{k, k}[\mathbf{I}]$ are normalized by the total number of co-occurrences.

For example, let us consider the pair of color component images $\left\{I_{\text {que }}^{k} ; I_{\text {tar }}^{k}\right\}$ represented by tables 1 and 2 , from which we evaluate the pair of co-occurrence matrices $\left\{M^{k, k}\left[\mathbf{I}_{\mathbf{q u e}}\right]\right.$; $\left.M^{k, k}\left[\mathbf{I}_{\text {tar }}\right]\right\}$ given in tables 3 and 4 , respectively. The spatial ranks of the levels in the color component images $I_{q u e}^{k}$ and $I_{\text {tar }}^{k}$ computed by means of equation (2) are given in tables 5 and 6 , respectively.

\begin{tabular}{|l|l|l|}
\hline 1 & 1 & 5 \\
\hline 1 & 2 & 4 \\
\hline 5 & 5 & 1 \\
\hline
\end{tabular}

\begin{tabular}{|l|l|l|}
\hline 11 & 11 & 15 \\
\hline 11 & 12 & 11 \\
\hline 15 & 15 & 15 \\
\hline
\end{tabular}

Table 1. Color component image $I_{q u e}^{k}$.

\begin{tabular}{|c|c|c|c|c|}
\hline$u \backslash v$ & $\mathbf{1}$ & $\mathbf{2}$ & $\mathbf{4}$ & $\mathbf{5}$ \\
\hline $\mathbf{1}$ & 6 & 4 & 2 & 4 \\
\hline $\mathbf{2}$ & 4 & 0 & 1 & 3 \\
\hline $\mathbf{4}$ & 2 & 1 & 0 & 2 \\
\hline $\mathbf{5}$ & 4 & 3 & 2 & 2 \\
\hline
\end{tabular}

Table 3. Co-occurrence matrix $M^{k, k}\left[\mathbf{I}_{\text {que }}\right]$ of table 1 .

Table 4. Co-occurrence matrix $M^{k, k}\left[\mathbf{I}_{\mathbf{t a r}}\right]$ of table 2 .

\begin{tabular}{|l|c|c|c|c|}
\hline$l$ & $\mathbf{1}$ & $\mathbf{2}$ & $\mathbf{4}$ & $\mathbf{5}$ \\
\hline$S R^{k}\left[\mathbf{I}_{\text {que }}\right](l)$ & $\frac{6}{40}$ & $\frac{14}{40}$ & $\frac{20}{40}$ & $\frac{40}{40}$ \\
\hline
\end{tabular}

Table 5. Spatial ranks of the levels within the color component image $I_{q u e}^{k}$ of table 1 . 


\begin{tabular}{|l|c|c|c|}
\hline$l$ & $\mathbf{1 1}$ & $\mathbf{1 2}$ & $\mathbf{1 5}$ \\
\hline$S R^{k}\left[\mathbf{I}_{\mathbf{t a r}}\right](l)$ & $\frac{8}{40}$ & $\frac{16}{40}$ & $\frac{40}{40}$ \\
\hline
\end{tabular}

Table 6. Spatial ranks of the levels within the color component image $I_{t a r}^{k}$ of table 2.

\subsection{Spatial ranks of sensor responses}

With the same manner, we define the spatial rank $\mathcal{S} \mathcal{R}\left(x^{k}(P)\right)$ of the CCD sensor response $x^{k}(P)$ to the color stimulus reflected by the surface which is projected onto the pixel $P$.

We consider two similar color images $\mathbf{I}_{\text {que }}$ and $\mathbf{I}_{\text {tar }}$, i.e. two images which represent the same object and which have been acquired under different illuminations. As most of invariant approaches do, we assume that the object can be translated or rotated only in a plane perpendicular to the optical axis of the camera, that is to say that all the elementary surfaces projected onto the pixels of $\mathbf{I}_{\mathbf{q u e}}$ are also projected onto pixels of $\mathbf{I}_{\mathbf{t a r}}$. Furthermore, since the images are similar, the pixels on which are projected the same elementary surfaces within the two images are spatially arranged with the same manner, so that two elementary surfaces projected onto two neighboring pixels in the query image are also projected onto two neighboring pixels in the target image. Consequently, we assume that the spatial ranks of the CCD sensor responses are preserved in case of illumination changes.

On the other hand, when the two considered images are different, i.e. they represent different objects, the spatial ranks of the sensor responses are not preserved.

Thus, we are able to discriminate the cases when the images are different from the case when they are similar thanks to the analysis of these spatial ranks. Since we cannot exactly determine the spatial ranks of the CCD sensor responses from a color image, we propose to estimate their probabilities of occurrence by introducing the fuzzy spatial rank concept.

\section{FUZZY SPATIAL RANKS}

\subsection{Sensor responses and component levels}

Under Lambertian assumptions, the sensor response $x^{k}(P)$, $k \in\{R, G, B\}$, to a color stimulus reflected by the elementary surface observed by the camera and projected onto the pixel $P$ depends on the spectral power distribution $E(\lambda)$ of the incident illuminant, on the spectral reflectance $\beta(P, \lambda)$ of the elementary surface projected onto $P$ and on the three spectral sensitivity functions $S^{k}(\lambda), k \in\{R, G, B\}$, of the camera sensors, so that :

$$
x^{k}(P)=\int_{\lambda} S^{k}(\lambda) \beta(P, \lambda) E(\lambda) d \lambda, k \in\{R, G, B\} .
$$

The sensor response $x^{k}(P)$ is quantified by the electronic device of the camera into $L$ levels to provide $c^{k}(P)$, the $k^{\text {th }}$ color component level of $P$ thanks to the analog-digital converter function $f[3]$ :

$$
c^{k}(P)=f\left(x^{k}(P)\right) .
$$

In this paper, we assume that the function $f$ is a monotonic increasing function, so that :

$$
\text { if } x^{k}\left(P^{\prime}\right)>x^{k}(P) \text { then } c^{k}\left(P^{\prime}\right) \geq c^{k}(P) \text {. }
$$

Unfortunately, we cannot exactly determine $x^{k}(P)$ from the color component level $c^{k}(P)$. However, since we assume that $f$ is a monotonic increasing function, we are only able to approximate the spatial ranks of the CCD sensor responses from the color component levels in the image.

Within a color component image $I^{k}$, let us denote $\left\{S R_{1}^{k}\right.$, $\left.S R_{2}^{k}, \ldots, S R_{N^{k}}^{k}\right\}$ the subset of $N^{k}$ successive spatial ranks of the color component levels sorted by the increasing order so that $S R_{i-1}^{k}<S R_{i}^{k}$.

Since $f$ is assumed to be a monotonic increasing function, we can show that the spatial rank $\mathcal{S} \mathcal{R}^{k}[\mathbf{I}]\left(x^{k}(P)\right)$ of the sensor response corresponding to the pixel $P$ in the color component image $I^{k}$ ranges between $S R_{i-1}^{k}$ and $S R_{i}^{k}$ when the spatial rank $S R^{k}[\mathbf{I}]\left(c^{k}(P)\right)$ of the color component level $c^{k}(P)$ within $I^{k}$ is equal to $S R_{i}^{k}$ :

$\left.\left.S R^{k}[\mathbf{I}]\left(c^{k}(P)\right)=S R_{i}^{k} \Rightarrow \mathcal{S R}^{k}[\mathbf{I}]\left(x^{k}(P)\right) \in\right] S R_{i-1}^{k} ; S R_{i}^{k}\right]$.

We propose to estimate the spatial ranks $\mathcal{S} \mathcal{R}^{k}[\mathbf{I}]\left(x^{k}(P)\right)$ of the sensor responses $x^{k}(P)$ from the spatial ranks $S R^{k}[\mathbf{I}]\left(c^{k}(P)\right)$ of the color component levels $c^{k}(P)$ thanks to fuzzy functions.

\subsection{Fuzzy spatial rank}

We associate with each spatial rank $S R_{i}^{k}$ a fuzzy subset composed by all the possible spatial ranks of sensor responses ranging between 0 and 1 . We define the membership degree $\mu_{S R_{i}^{k}}^{k}(\mathcal{S R})$ of a spatial rank $\mathcal{S R}$ of a sensor response to this subset as:

$$
\left\{\begin{array}{l}
\left.\left.\mu_{S R_{i}^{k}}^{k}(\mathcal{S R})=\frac{1}{S R_{i}^{k}-S R_{i-1}^{k}} \text { if } \mathcal{S R} \in\right] S R_{i-1}^{k} ; S R_{i}^{k}\right] \\
\mu_{S R_{i}^{k}}^{k}(\mathcal{S R})=0 \text { else. }
\end{array}\right.
$$

As no prior knowledge is available about the distribution of spatial ranks $\mathcal{S R}$ of sensor responses within their associated spatial rank intervals, we assume that they are equiprobably spread.

\subsection{Histogram of fuzzy spatial ranks}

When the fuzzy spatial ranks of the sensor responses have been estimated from the image, we propose to characterize the image by the histogram of fuzzy spatial ranks. Since this 
estimation is based on the spatial ranks of the color component levels, we can deduce the histogram $\eta$ of fuzzy spatial ranks of sensor responses from the histogram $\mathcal{H}$ of spatial ranks of color component levels as :

$$
\begin{aligned}
\eta[\mathbf{I}]\left(\mathcal{S} \mathcal{R}^{R}, \mathcal{S} \mathcal{R}^{G}, \mathcal{S} \mathcal{R}^{B}\right)= & \\
\sum_{u=1}^{N^{R}} \sum_{v=1}^{N^{G}} \sum_{w=1}^{N^{B}} \mu_{S R_{u}^{R}}^{R}\left(\mathcal{S} \mathcal{R}^{R}\right) & \mu_{S R_{v}^{G}}^{G}\left(\mathcal{S \mathcal { R } ^ { G }}\right) \mu_{S R_{w}^{B}}^{B}\left(\mathcal{S} \mathcal{R}^{B}\right) \\
& \times \mathcal{H}[\mathbf{I}]\left(S R_{u}^{R}, S R_{v}^{G}, S R_{w}^{B}\right),
\end{aligned}
$$

with $\mathcal{S R}^{k} \in\left\{0, \frac{1}{M}, \ldots, \frac{M}{M}\right\}, k \in\{R, G, B\}$.

For the implementation purpose, the spatial ranks $\mathcal{S} \mathcal{R}$ of sensor responses are quantified with $(M+1)$ levels, $M$ being adjusted by the analyst, so that $\mathcal{S} \mathcal{R}=0, \frac{1}{M}, \frac{2}{M}, \ldots, \frac{M}{M}$.

\section{EXPERIMENTAL RESULTS}

\subsection{Object recognition across illumination changes with the SFU database}

We propose to demonstrate the improvement of the intersection between the pairs of histograms of fuzzy spatial ranks for object recognition purpose across illumination changes. We use the Simon Fraser University (SFU) database [2] available at http://www.cs.sfu.ca/ colour/data. Its 187 images contain 17 objects lit by one of 11 available illumination sources and acquired with the same viewing conditions by one camera (see figure 2).

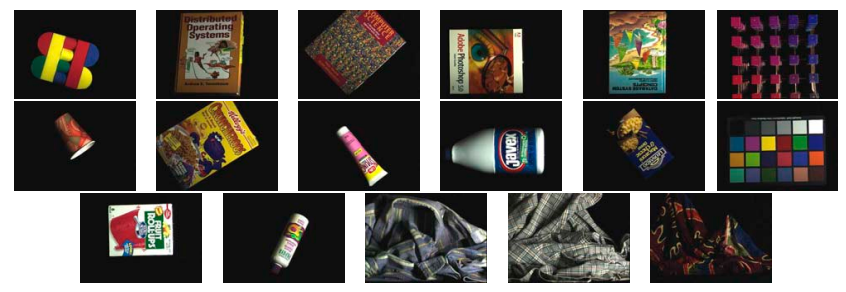

Fig. 2. The 17 objects of the SFU database.

For object searching, the images acquired under one illumination, called the target illumination, are considered as being the target images and one of those acquired under one of the 10 other illumination sources, called the query illumination, is considered as being the query image. So, there are $11 \times 10$ different pairs of query-target illumination. The image retrieval is repeated for each of the 17 objects. Finally, 1870 retrievals are achieved $(17$ objects $\times 11 \times 10$ pairs of different illumination).

For each image retrieval, the 17 target images are ordered with respect to the intersections between their invariant color histograms and the invariant color histogram of the considered query image. When the first ordered target image is similar to the query image, the research result is considered as perfect.
We propose to compare the results obtained by intersection between the histograms of the color component levels ranks [3] with those obtained by the intersection between the histograms of the fuzzy spatial ranks.

Each column of table 7 indicates the percentage of successful image retrievals.

\begin{tabular}{|c|c|c|c|}
\hline $\begin{array}{c}\text { Intersection } \\
\text { between }\end{array}$ & $(M=16)$ & $(M=64)$ & $(M=256)$ \\
\hline $\begin{array}{c}\text { histograms of } \\
\text { ranks }\end{array}$ & 89.89 & 75.08 & 48.72 \\
\hline $\begin{array}{c}\text { histograms of } \\
\text { fuzzy spatial } \\
\text { ranks }\end{array}$ & 97.81 & 92.83 & 88.24 \\
\hline
\end{tabular}

Table 7. Object recognition results obtained by the intersections between different histograms with the SFU database.

Table 7 shows that, for object recognition across illumination changes, the intersection between the histograms of fuzzy spatial ranks provides better results than those obtained by the intersection between histograms of ranks, for significantly different values of $M$. Furthermore, Table 7 shows that the quality of object recognition by the intersection between the histograms of ranks is very sensitive to $M$. On the other hand, the results obtained by the intersection between the histograms of fuzzy ranks remain stable when $M$ varies.

\section{REFERENCES}

[1] B.V. Funt, K. Barnard, and L. Martin, "Is machine colour constancy good enough?," in Procs. of the $5^{\text {th }}$ European Conf. on Computer Vision, 1998, pp. 445-459.

[2] G.D. Finlayson and G. Schaefer, "Colour indexing across devices and viewing conditions," in Procs. of the $2^{\text {nd }}$ Int. Workshop on Content-Based Multimedia Indexing, Brescia, Italy, 2001, pp. 215-221.

[3] G. Finlayson, S. Hordley, G. Schaefer, and G. Y. Tian, "Illuminant and device invariant colour using histogram equalisation," Pattern Recognition, vol. 38, pp. 179-190, 2005.

[4] D. Muselet, L. Macaire, and J.G. Postaire, "Color histograms adapted to query-target images for object recognition across illumination changes," EURASIP Journal on Applied Signal Processing, Special Issue on Advances in Intelligent Vision Systems : Methods and Applications, vol. 14, pp. 2164-2172, 2005. 